\title{
ACUTE ANTERIOR UVEITIS AFTER ZOLEDRONIC ACID INFUSION FOR OSTEOPOROSIS TREATMENT
}

Lucas Guimarães Nolasco Farias ${ }^{1, *}$, Bruno Batista da Cunha Filho ${ }^{1}$, Amanda Bernardes ${ }^{1}$, Sofia Silveira de Castro Miranda ${ }^{1}$, Paulo Guilherme de Oliveira Barbalho Junior ${ }^{1}$, Elaine de Azevedo ${ }^{1}$, Rina Dalva Neubarth Giorgi ${ }^{1}$

1.Instituto de Assistência Médica ao Servidor Público Estadual, São Paulo (SP), Brazil.

*Corresponding author: lucasguimaraes1995@hotmail.com

\section{BACKGROUND}

Bisphosphonates are synthetic analogues of organic pyrophosphate, an endogenous inhibitor of bone mineralization. Antiresorptive effects are manifested through inhibition of osteoclast function, in addition to the formation and dissolution of hydroxyapatite crystals. They are widely used for treatment of osteometabolic disorders, such as osteoporosis, Paget's disease and hypercalcemia of malignancy. The most used bisphosphonates in clinical practice are alendronate, risedronate, pamidronate and zoledronic acid. There are side effects to this class of drugs, including flu-like syndrome, nausea, bone pain, hypocalcemia and jaw osteonecrosis. There are few reports of ocular complications in the literature, mostly associated with alendronate and pamidronate, and rarely with zoledronic acid.

\section{CASE REPORT}

A 54-year-old female patient with osteoporosis diagnosed in 2020, who received the first dose of intravenous zoledronic acid. She had no history of ophthalmic pathologies or allergies. She developed fever, arthralgia and myalgia $24 \mathrm{~h}$ after the infusion. Within $48 \mathrm{~h}$, there were visual turbidity, erythema and ocular pain bilaterally. She was evaluated in an ophthalmology service and had an intraocular pressure of $14 \mathrm{mmHg}, 20 / 40$ visual acuity, anterior chamber reaction and pigments deposited in endothelium and anterior capsule of both eyes. There was no evidence of retinal or vitreous involvement. She was diagnosed with acute anterior uveitis associated with zoledronic acid and received a prescription for steroid and atropine-based eye drops. The condition was completely resolved after 6 weeks of treatment. She did not receive new doses after this event.

\section{CONCLUSION}

Acute uveitis associated with zoledronic acid is rare, occurring within 24 to $48 \mathrm{~h}$ of prescription. Physicians who prescribe intravenous bisphosphonate should be aware of that complication and instruct patients to search for ophthalmologic evaluation in case of development of ocular symptoms after infusion.

\section{KEYWORDS}

Uveitis, Zoledronic acid, Bisphosphonate. 